\title{
Real-Time Multiobjective Microgrid Power Management Using Distributed Optimization in an Agent-Based Bargaining Framework
}

\section{Authors: Keveh Dehghanpour and Hashem Nehrir}

(c) 2018 IEEE. Personal use of this material is permitted. Permission from IEEE must be obtained for all other users, including reprinting/ republishing this material for advertising or promotional purposes, creating new collective works for resale or redistribution to servers or lists, or reuse of any copyrighted components of this work in other works.

Dehghanpour, Kaveh, and Hashem Nehrir. "Real-Time Multiobjective Microgrid Power Management Using Distributed Optimization in an Agent-Based Bargaining Framework." IEEE Transactions on Smart Grid 9, no. 6 (November 2018): 6318-6327. doi:10.1109/ tsg.2017.2708686. 


\title{
Real-Time Multiobjective Microgrid Power Management Using Distributed Optimization in an Agent-Based Bargaining Framework
}

\author{
Kaveh Dehghanpour, Student Member, IEEE, Hashem Nehrir, Life Fellow, IEEE,
}

\begin{abstract}
In this paper, we propose a Multi-Objective Power Management (MOPM) procedure for MicroGrids (MG). Through this procedure the power management problem is modeled as a bargaining game among different agents with different sets of objective functions. Nash Bargaining Solution (NBS) is employed to find the solution of the bargaining game. NBS lies on the Pareto-front of the power management problem. Moreover, it introduces a unique and fair balance among the objective functions of different agents and removes the need to track the whole Pareto-front in real-time. Distributed Gradient Algorithm (DGA) is applied to find the NBS through a modular distributed decision framework without using a central control unit. In this way, the problem of data privacy of different parties within the MG is addressed. The proposed methodology has been tested through simulations on islanded and grid-connected MGs under different pricing scenarios (fixed versus Time-of-Use (ToU) pricing).
\end{abstract}

Index Terms-Microgrids, distributed optimization, agentbased modeling, power management.

\section{NOMENCLATURE}

$\begin{array}{ll}a, b, c & \begin{array}{l}\text { Coefficients of the quadratic cost function of } \\ \text { the DG unit. }\end{array} \\ a_{i}^{j} & \begin{array}{l}\text { Processing weight of the data received from the } \\ j^{t h} \text { agent in the } i^{t h} \text { agent decision model. }\end{array} \\ d_{i} & \text { Disagreement point of the } i^{t h} \text { agent. } \\ E_{C} & \text { Fuel energy density of the DG unit. } \\ E_{\max } & \text { Maximum energy capacity of the battery bank. } \\ E_{S c} & \text { Total scheduled energy consumption of time- } \\ & \text { shiftable loads. } \\ f_{i} & \text { Objective function of the } i^{t h} \text { agent. } \\ G R C & \text { Generation Rate Constraint. } \\ H & \text { Number of time steps in the decision window. } \\ M & \text { Number of elements of } P . \\ N & \text { Total Number of agents. } \\ N_{i} & \text { Number of neighboring agents for the } i^{t h} \\ & \text { agent. } \\ O_{i} & \text { Number of objective functions for the } i^{t h} \\ P & \text { agent. } \\ P_{C} & \text { Power management decision vector. } \\ P_{C}^{\max } & \text { Aggregated curtailed load power. } \\ P_{C}^{\min } & \text { Maximum curtailable load limit. } \\ P_{D G} & \text { Minimum curtailable load limit. } \\ P_{D G}^{\max } & \text { Power output of DG unit. } \\ & \text { Maximum DG power boundary. }\end{array}$

K. Dehghanpour, and H. Nehrir are with the Department of Electrical and Computer Engineering, Montana State University, Bozeman, MT 59717 USA (e-mail: Kaveh.dehghanpour@msu.montana.edu; hnehrir@montana.edu).

\begin{tabular}{|c|c|}
\hline$P_{D G}^{\min }$ & Minimum DG power boundary. \\
\hline$P_{E S S}$ & Output power of the battery system. \\
\hline$P_{E S S}^{\max }$ & Maximum battery power boundary. \\
\hline$P_{E S S}^{\min }$ & Minimum battery power boundary. \\
\hline$P_{f}$ & Value of total fixed load. \\
\hline$P_{G}$ & Imported power from the main grid. \\
\hline$P_{G}^{\max }$ & Maximum power import limit. \\
\hline$P_{G}^{\min }$ & Minimum power import limit. \\
\hline$P_{R}$ & Renewable PV power. \\
\hline$P_{S c}$ & $\begin{array}{l}\text { Scheduled aggregate power of time-shiftable } \\
\text { loads. }\end{array}$ \\
\hline$P_{T S}$ & Aggregate power of time-shiftable loads. \\
\hline$S O C$ & SOC of the battery system. \\
\hline$S O C^{\max }$ & Maximum SOC limit. \\
\hline$S O C^{\min }$ & Minimum SOC limit. \\
\hline$T$ & Length of the decision horizon. \\
\hline$t$ & Time instant. \\
\hline$t_{\max }$ & $\begin{array}{l}\text { Maximum allowable employment time for } \\
\text { time-shiftable loads. }\end{array}$ \\
\hline$t_{\min }$ & $\begin{array}{l}\text { Minimum allowable employment time for } \\
\text { time-shiftable loads. }\end{array}$ \\
\hline$u_{i}$ & Utility function of the $i^{t h}$ agent. \\
\hline$X_{i}$ & Feasible decision region of the $i^{t h}$ agent. \\
\hline$x_{i}$ & Decision vector of the $i^{t h}$ agent. \\
\hline$\alpha_{k}$ & Time-varying weight factor. \\
\hline$\gamma_{i}^{j}$ & $\begin{array}{l}\text { Weight variables for the weighted-sum central } \\
\text { optimization model. }\end{array}$ \\
\hline$\Delta t$ & Time step. \\
\hline$\lambda_{F}$ & Unit price of the fuel for the DG unit. \\
\hline$\lambda_{G}$ & Unit price of the main grid electricity. \\
\hline$\omega$ & $\begin{array}{l}\text { Collective flexibility coefficient of DR re- } \\
\text { sources. }\end{array}$ \\
\hline
\end{tabular}

\section{INTRODUCTION}

$\mathbf{T}$ He growth of renewable energy resources in the form of distributed on-site generation has led to the introduction of the concept of MicroGrid (MG) [1]. An MG is basically a small-scale cluster of different types of controllable and uncontrollable microsources connected together at electrical distribution level. These microsources include on-site generators (both Variable Renewable Sources (VRS) and nonrenewable Dispatchable Generators (DG)), Energy Storage Systems (ESS), and fixed and controllable loads (i.e., Demand Response (DR)) [2]. Each MG can be thought of as a selfsustainable energy unit with its own decision-making and 
control capabilities, which is able to continue operating and serving consumers even when it is islanded from the main grid. Hence, MGs not only enable the penetration of renewable energy resources at the electrical distribution level, but also introduce some level of "resiliency" into the power grid due to their islanding capability [3].

In order to utilize an MG, a hierarchical control and decision-making structure is required [4]. At the lower level of this hierarchy each microsource within the MG employs a local control unit (which is usually based on droop characteristics [5]). At the higher level of the hierarchy the local controllers are coordinated by a power management process to reach a desirable global behavior throughout the MG. Basically, the power management apparatus provides reference signals for the lower-level control systems. In this paper we propose and investigate a novel agent-based power management procedure which can be applied to both gridconnected and islanded MGs.

Most of the power management procedures proposed in the literature are aimed at single-objective optimization, namely, total operational cost minimization [6] or power loss minimization [7]. However, in general, the power management problem within an MG can have several competing objective functions. Hence, different microsources of an MG can have different objectives or sets of objective functions. Compared to a single-objective approach, the proposed Multi-Objective (MO) optimization formulation allows us to reach trade-off solutions among several competing objective functions (e.g., cost savings/comfort level, efficiency/profit) and explicitly consider the effect of different objective functions within system operation. The goal of Multi-Objective Power Management (MOPM) optimization problem of MGs is to find the set of non-dominated optimal trade-off solutions, known as the Pareto-front of the optimization problem [8]. In [9] a MO linear programming approach is proposed for MG power management using multi-layered Artificial Neural Networks (ANN). Another MOPM technique is introduced in [10] employing Non-dominated Sorting Genetic Algorithm (NSGAII) to obtain the Pareto-front of the optimization problem. In [11], different objective functions are also considered in energy management of an $\mathrm{MG}$ with high penetration of renewable power sources, through valuation functions. In another work, the Pareto-front of the objective functions of an $\mathrm{MG}$ is obtained using a sampling approach and an agent-based model [12]. Also, in [13] the MOPM problem of MGs is modeled and solved as a static non-cooperative game.

While in the previous works the whole Pareto-front is obtained through sampling, point-by-point optimization, or evolutionary population-based algorithms, these approaches can be numerically burdensome for real-time applications as the number of objective functions increases. Hence, to address this problem, in this paper we propose a power management approach that is able to find a "unique" and "fair" solution to the MOPM problem of MGs without the need to track the whole Pareto-front in real-time. A fair solution strategy should not discriminate between the objectives (i.e., under symmetric conditions it should yield identical results for different objectives). The proposed power management procedure is based on the idea of Nash Bargaining Solution (NBS) [14]. Hence, The MO optimization problem can be viewed as a bargaining game consisting of different agents (players) with different sets of objective functions. Each controllable microsource within the MG is assigned an agent which participates in the bargaining process on behalf of the parties in charge of the microsources. Thus, MG is modeled as a cooperative community of producers and consumers, where each entity is interested in obtaining a trade-off between its available objective functions and the objective functions of other agents (while maintaining the overall cooperative nature of the decision problem). NBS is selected as a solution strategy since it was designed to address situations of cooperative decision making with bargaining, which is essential in the case of an MG power management model.

Due to its advantages, NBS has been used in different industry-related applications with the goal of fair resource allocation; a few examples are as follows: bandwidth, subcarrier, and power allocation along with relay cooperation in communication systems [15] [16] [17], developing licensing policies [18], designing permit allocation rules for pollution control [19], marriage market and household labor studies [20], and traffic control [21].

The NBS of a bargaining game lies on the Pareto-front of the pay-off functions (i.e., objective functions) of the agents in the game. Apart from its uniqueness, NBS introduces a fair balance among the objective functions of the agents. We have shown in [22] that the NBS of the MOPM problem of an MG can be computed using a central optimization method. In this paper, we will demonstrate that the NBS of the bargaining game can be obtained through a Distributed Sum Optimization (DSO) problem [23] which can be solved using an agent-based platform via a distributed optimization technique. The employed solution technique is known as Distributed Gradient Algorithm (DGA) [23]-[25], which was selected due to its ease of implementation and its desirable mathematical properties. Using DGA different control agents (with different objective functions) within a communication network are able to interact and exchange data with each other to solve a DSO problem, cooperatively, without a central solver. While DGA has already been applied for solving power engineering optimization problems [26], its use has been limited to single-objective optimization problems. In this paper, we extend DGA to a distributed bargaining situation to solve the MOPM problem of MGs. Thus, unlike previous MOPM methods in the literature, the proposed approach is modular and distributed by nature. In this way, a distributed bargaining framework is designed through which the data privacy of different parties within the system is respected (i.e., to reach a solution, the agents do not need to share their private cost/utility and feasible decision region with each other). Also, by removing a central control unit, we are basically removing a single point of failure. This implies that a more secure system automation with plug-and-play capabilities can be achieved through the proposed distributed optimization framework. Using a distributed reasoning approach, we obtain an inter-operable system with plug-and-play capabilities [27]. This bargaining framework enables the agents to reach a 


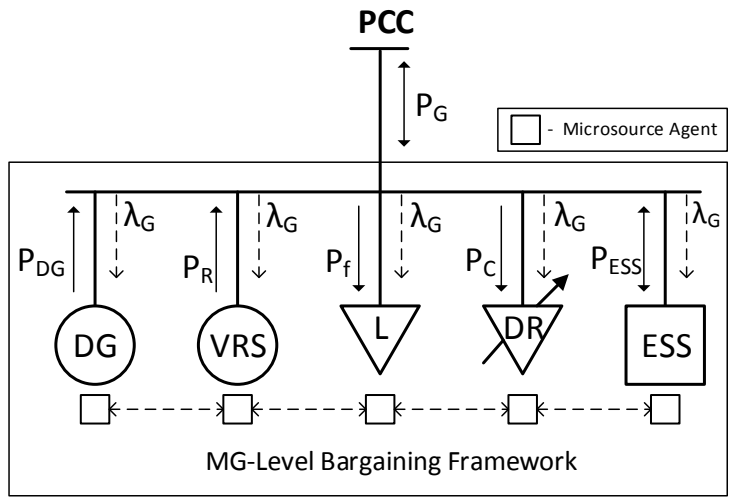

Fig. 1. Generic MG architecture.

fair agreement which lies on the Pareto-front of the MOPM problem. To demonstrate the efficiency of the framework, the proposed method is tested on both islanded and grid-connected MGs through numerical simulations under fixed and Time-ofUse (ToU) pricing scenarios.

To summarize, the contributions of the paper are as follows:

- Using game theory, the MG is modeled as a community of cooperative agents with competing objective functions that bargain with each other to reach a fair and Paretoefficient outcome.

- The bargaining process, which is basically the MOPM of the MG, is solved using the concept of NBS, which provides desirable properties: uniqueness, fairness, and Pareto-efficiency.

- The computational process is performed through an agent-based distributed optimization framework, without a central control unit. Employing this framework, the agents are able to reach the NBS, without sharing their private cost and constraint data.

The rest of the paper is constructed as follows: in Section II the overall structure of the MOPM problem of MGs is discussed. The distributed bargaining/optimization framework is designed in Section III. The results of the numerical experiments are shown and discussed in Section IV. The conclusions of the paper are presented in Section V.

\section{MG Power Management Problem}

In this section the agent-based MOPM problem is discussed in details. A generic MG consisting of different microsources is shown in Fig. 1. Each microsource is equipped with a control agent which participates in the MG-based bargaining framework on behalf of the element. The microsources considered in this paper are: thermal DG unit, battery storage bank, DR resources (consisting of curtailable and timeshiftable loads), Photo-Voltaic (PV) power generator, and fixed uncontrollable loads. Note that the control agents within the MG are "price-takers" (due to their relatively small size and low market share). Hence, the price signals (both the fixed and ToU tariffs) are the input variable to the decision model, which are set by a power utility company.

\section{A. Timing Strategy and Uncertainty Handling}

In this paper we have adopted a rolling horizon optimization strategy [6]: at each time instant $(t)$ the power management problem is solved for a certain look-ahead time $(t+T)$, based on forecasted values for renewable energy resources and the load. The decision horizon is divided into $H$ time steps $(\Delta t)$. The goal of the power management problem is to optimize the decision variables (i.e., output power of the controllable microsources) at different time steps of the decision window. After the power management problem is solved, the values of the decision variables corresponding to the immediate time step $(t+\Delta t)$ are sent to the local control devices of the microsources. The values of the decision variables for the rest of the decision horizon can be stored as back-up or be used as initial conditions in future rounds of the power management optimization (i.e., hot start). Then the decision window is rolled one step ahead (i.e., $t:=t+\Delta t$ ).

As discussed above, the values of certain variables (fixed load and PV power) need to be forecasted and used in the power management problem. The prediction error is a source of uncertainty in the decision making. In this paper, two such sources of uncertainty are considered in the modeling: prediction error of renewable energy sources and load prediction error. These errors are represented by Gaussian distribution functions which are adopted from [28] and [29]. Note that the distribution of forecasting error changes at different time steps of the decision horizon. Hence, as the decision window rolls through time the forecasted values of the load and renewable power are also updated based on the time-varying distribution functions of the prediction error.

\section{B. Problem Formulation}

The objective functions and constraints of different microsources considered in the power management problem are discussed in this subsection. The objective functions are denoted as $U_{i}^{j}$, indicating the $j^{\text {th }}$ objective of the $i^{\text {th }}$ microsource.

1) DG unit: Two objective functions are considered for the DG unit: total profit (i.e., total revenue less total cost) from local power generation $\left(U_{1}^{1}\right)$ and average efficiency $\left(U_{1}^{2}\right)$.

$$
\begin{gathered}
U_{1}^{1}=\sum_{t=1}^{H}\left\{-P_{G}(t) \lambda_{G}(t)-\left(a \cdot P_{D G}(t)^{2}+b \cdot P_{D G}(t)+c\right)\right\} \\
U_{1}^{2}=\frac{1}{H} \cdot \sum_{t=1}^{H}\left\{\frac{k \cdot P_{D G}(t)}{a \cdot P_{D G}(t)^{2}+b \cdot P_{D G}(t)+c}\right\}
\end{gathered}
$$

where, coefficients $a, b$, and $c$ define the quadratic cost function of the DG, according to [30]. Objective function (1) is composed of two terms: the cost/revenue of power exchange with the grid and the cost of local power generation using the DG unit. Hence, this objective function defines the profitability of power production 
from the DG unit. The reason that the DG control agent is considered to be in charge of this objective is that this agent has direct access to DG cost data. Note that depending on the sign of the power exchanged with the grid at the PCC, the first term in objective function (1) (i.e., $-\lambda_{G} P_{G}$ ) can be positive or negative (i.e., $P_{G} \geq 0$ implies power import from the grid, and $P_{G} \leq 0$ implies power export to the grid). Hence, the first term in objective function (1) can be both revenue (for $P_{G} \leq 0$ ) or cost (for $P_{G} \geq 0$ ). When the DG is isolated from the main grid, since the revenue from sales of power to grid is eliminated, $U_{1}^{1}$ turns into the negative cost of production. The efficiency equation (2) is also adopted from [30], along with the expression for the coefficient $k$ used in (2), defined as:

$$
k=\frac{3600 \cdot \lambda_{F}}{E_{C}}
$$

The constraints on the DG unit are as follows:

$$
\begin{gathered}
P_{D G}^{\min } \leq P_{D G}(t) \leq P_{D G}^{\max } \\
\frac{\left|P_{D G}(t)-P_{D G}(t-1)\right|}{\Delta t} \leq G R C
\end{gathered}
$$

where, (4) gives the production limits on output power of the DG. The Generation Rate Constraint (GRC) is enforced by (5). This constraint defines the ramp rate limit of the thermal DG unit.

2) ESS unit: In this paper, no objective function is considered for the battery storage system. However, the following constraints are taken into account:

$$
\begin{gathered}
P_{E S S}^{\min } \leq P_{E S S}(t) \leq P_{E S S}^{\max } \\
S O C(t)=S O C(t-1)-\frac{\Delta t}{E_{\max }} \cdot P_{E S S}(t) \\
S O C^{\min } \leq S O C(t) \leq S O C^{\max }
\end{gathered}
$$

where, positive and negative values for $P_{E S S}$ define discharging and charging states, respectively. The normalized State Of Charge (SOC) of the battery is determined as (7) and maintained within its boundaries $\left(\left[S O C^{\min }, S O C^{\max }\right]\right)$. Note that the power losses of the battery system are ignored, which is reasonable for high energy efficiency battery systems.

3) Demand Response Provider (DRP) unit: The DRP is a load aggregator in charge of providing DR services in the MG, by controlling the collective controllable loads. Two types of controllable load resources are considered in this paper: curtailable loads and time-shiftable loads.

- Curtailable loads: At certain times, some percentage of the total demand is available for curtailment. The curtailment process can cause discomfort to the users and is to be minimized. Hence, a concave utility function $\left(U_{2}^{1}\right)$ is defined to penalize deviation from the desired consumption schedule. An exponential utility function is adopted here based on [31].

$$
\begin{gathered}
U_{2}^{1}=\frac{1}{H} \cdot \sum_{t=1}^{H}\left\{\lambda_{G}(t) \cdot P_{f}(t) \cdot\left(1-e^{-\omega\left(P_{f}(t)-P_{C}(t)\right)}\right)\right\} \\
P_{C}^{\min } \leq P_{C}(t) \leq P_{C}^{\max }
\end{gathered}
$$

where, constraint (10) defines the boundaries of the aggregate curtailable load power, which is between its minimum and maximum values (i.e., $\left.\left[P_{C}^{\min }, P_{C}^{\max }\right]\right)$.

- Time-shiftable loads: This type of DR resource is composed of the aggregation of loads that need to consume a certain amount of energy within a certain time frame $\left[t_{\min }, t_{\max }\right]$ (e.g., electric vehicles' charging load). Hence, such loads cannot be employed sooner than $t_{\min }$ or later than $t_{\max }$. Similar to curtailable loads, a concave utility function $\left(U_{2}^{2}\right)$ is defined for time-shiftable loads to penalize deviations from the pre-defined customer schedule:

$$
\begin{gathered}
U_{2}^{2}=\sum_{t=1}^{H}\left\{-\left(P_{T S}(t)-P_{S c h}(t)\right)^{2}\right\} \\
P_{T S}^{\min } \leq P_{T S}(t) \leq P_{T S}^{\max }, \forall t \in\left[t_{\min }, t_{\max }\right] \\
\sum_{t=t_{\min }}^{t_{\max }} P_{T S}(t) \cdot \Delta t=\sum_{t=t_{\min }}^{t_{\max }} P_{S c h}(t) \cdot \Delta t=E_{S c h}
\end{gathered}
$$

while (12) enforces the maximum/minimum limits of time-shiftable DR resource, (13) maintains and preserves the total scheduled energy consumption $\left(E_{S c h}\right)$. Note that these constraints also guarantee that the time-shiftable resources are employed within the $\left[t_{\min }, t_{\max }\right]$ interval.

Cost saving is considered to be the third objective function of the DRP, denoted as $U_{2}^{3}$ :

$$
U_{2}^{3}=\sum_{t=1}^{H}\left\{P_{T S}(t) \lambda_{G}(t)-P_{C}(t) \lambda_{G}(t)\right\}
$$

4) PCC link: To prevent overloading in the link that connects the MG with the main grid at the PCC and defer distribution system expansion/upgrades, the following objective function (adopted from [11]) and the corresponding constraint are used:

$$
\begin{gathered}
U_{3}^{1}=\sum_{t=1}^{H}\left\{-\left(P_{G}(t)\right)^{2}\right\} \\
P_{G}^{\min } \leq P_{G}(t) \leq P_{G}^{\max }
\end{gathered}
$$

where, $P_{G}^{\min }$ and $P_{G}^{\max }$ denote the limits for power export and import to/from the main grid, respectively. The idea behind objective function (15) is to promote self-sufficiency of the MG and reduce the reliance on the grid, while maintaining a safe margin to avoid congestion/overloading. Note that while constraint (16) enforces the physical congestion/overloading boundary 
at the PCC, it is not necessarily able to create a desired safe congestion margin. Another approach to this problem is to eliminate objective function (15) and tighten the constraint (16).

5) PV and fixed load: The renewable PV power $\left(P_{R}\right)$ and the fixed load power $\left(P_{f}\right)$ are assumed to be nondispatchable at all times. These powers, as explained in the previous subsection, are simply predicted (with a certain forecast error). While the PV power does not appear as decision variable in the power management problem, it is controlled at the lower level of the hierarchy using maximum power point tracking.

Apart from the private constraints of different resources discussed above, the global MG-wide power balance constraint, which connects the output power of all the power sources in the system, should also be respected at all times:

$P_{D G}(t)+P_{R}(t)+P_{E S S}(t)+P_{G}(t)+P_{C}(t)=P_{f}(t)+P_{T S}(t)$

Hence, the MOPM problem for the MG can be written as follows:

$$
\begin{gathered}
\max _{\boldsymbol{P}}\left\{U_{1}^{1}, U_{1}^{2}, U_{2}^{1}, U_{2}^{2}, U_{2}^{3}, U_{3}^{1}\right\}, \\
\text { s.t. (4), (5), (6), (7), (8), (10), (12), (13), (16), (17) }
\end{gathered}
$$

where, $\boldsymbol{P}$ is the vector of decision variables, consisting of the power of controllable microsources within the MG (including the exchanged power with the main grid) for the look-ahead time in which the power management problem is solved:

$$
\begin{aligned}
& \boldsymbol{P}=\left[P_{D G}(1), \ldots P_{D G}(H), P_{E S S}(1), \ldots P_{E S S}(H),\right. \\
& \left.P_{G}(1), \ldots P_{G}(H), P_{C}(1), \ldots P_{C}(H), P_{T S}(1), \ldots P_{T S}(H)\right]^{T}
\end{aligned}
$$

where, $[.]^{T}$ denotes vector transpose operation. In this paper vector quantities are indicated via bold letters. We also define $M$ to be the number of elements of the decision vector $\boldsymbol{P}$ for future use (i.e., $\boldsymbol{P} \in \mathbb{R}^{M}$ ). Note that in the optimization problem (18), all the objective functions are concave in the decision variable and the constraints form convex sets. Hence, the overall MOPM problem is convex, which implies that the Pareto-front defines the boundary of a convex set [8]. As will be discussed in the next section, convexity of the power management problem is crucial for the NBS to be welldefined. To solve this optimization problem a distributed agentbased bargaining framework is designed.

\section{Agent-BASED Distributed BARGaining FRAMEWORK}

In this section, first we formulate the MOPM problem as a bargaining game. Then a distributed optimization technique is employed to find the solution to the game.

\section{A. NBS as a Solution Concept}

A bargaining game is used to model a situation of interactive cooperative resource allocation among several agents. Hence, bargaining games consist of the following elements [14]:
- A set of $N$ players (agents), $A g=\{1, \ldots, N\}$ that negotiate with each other to find a globally agreeable solution.

- A set of utility or pay-off functions, $U=\left\{u_{1}, \ldots, u_{N}\right\}$, where $u_{i}$ denotes the $i^{\text {th }}$ player's pay-off level at each state of the bargaining game. The goal of each agent is to maximize its pay-off function through negotiations with its peers.

- A set of disagreement points, $D=\left\{d_{1}, \ldots, d_{N}\right\}$, where $d_{i}$ denotes the worst case pay-off value for the $i^{t h}$ player if the negotiations break down. Hence, the disagreement points represent the tacit threat of inability of agents to reach an agreement. If the bargaining game is welldefined (i.e., the disagreement points have worse performance than every other solution) and the agents are "rational" then the disagreement point is never reached.

The Pareto-front of the pay-off functions of the players constitute the set of all the rational solutions that the agents can reach through negotiations. Given that the Pareto-front is the optimal trade-off set of the pay-off space of the agents, any deviation from this set would be detrimental to at least one agent. In other words, the Pareto-front of the game determines the set of equilibria to the bargaining situation. NBS was proposed to define a single unique solution (among the candidates on the Pareto-front) to the bargaining game. The properties of NBS are as follows [14]:

- NBS can be obtained using optimization problem (20). This optimization problem is solved over the feasible solution set within the pay-off space of the players $\left(F_{U}\right)$.

$$
N B S=\underset{\left(u_{1}, \ldots, u_{N}\right) \in F_{U}}{\arg \max } \prod_{i=1}^{N}\left(u_{i}-d_{i}\right) .
$$

Optimization problem (20) can be formulated as follows:

$$
N B S=\underset{\left(u_{1}, \ldots, u_{N}\right) \in F_{U}}{\arg \max } \sum_{i=1}^{N} \log \left(u_{i}-d_{i}\right) .
$$

Compared to (20), (21) is much more beneficial for our purpose, as will become clear in the next subsection.

- Uniqueness: If $F_{U}$ is a convex set (i.e., the bargaining game is convex) then (21) is a convex optimization problem and NBS is the global optimum to the problem and is unique.

- Efficiency (Pareto-optimality): Given a convex bargaining game, NBS (obtained through (21)) is guaranteed to be Pareto-optimal (i.e., NBS lies on the Pareto-front of the bargaining game).

- Fairness: If the bargaining game is symmetric with respect to players $i$ and $j$ (i.e., if $\left(u_{i}=u, u_{j}=u^{\prime}\right) \in F_{U}$ then $\left.\left(u_{i}=u^{\prime}, u_{j}=u\right) \in F_{U}\right)$, then those players will receive the same pay-off value with NBS. This means that NBS does not discriminate between identical players and the outcome of the bargaining is guaranteed to be fair under NBS.

- Covariance under positive affine transformation: NBS is independent of unit of measurement of the pay-off functions of the players. Also, multiplying the objective 
functions by scalars does not change the outcome of the bargaining.

- Independence of irrelevant alternatives: NBS is insensitive to changes in the set of inferior alternative solutions.

Since in both MO optimization problem and bargaining game the goal is to reach a solution on the Pareto-front of the problem, the MOPM problem (18) can be thought of as a bargaining game, with each agent having different sets of objective functions. Since (18) is a convex optimization problem, NBS can be applied to find a unique, fair, and Pareto-optimal solution for the power management problem. Given (21), the NBS-based power management problem is formulated as follows:

$$
\begin{gathered}
\max _{\boldsymbol{P}}\left\{\sum_{i=1}^{N} \log \left(\prod_{j=1}^{O_{i}}\left(U_{i}^{j}-d_{i}^{j}\right)\right)\right\}, \\
\text { s.t. (4), (5), (6), (7), (8), (10), (12), (13), (16), (17) }
\end{gathered}
$$

where, $O_{i}$ denotes the number of objective functions considered for the $i^{t h}$ agent. Note that optimization problem (22) is also convex and has a unique globally optimal solution, which defines the NBS to the bargaining among the agents.

\section{B. Distributed Optimization Approach}

Our goal in this paper is to solve (22) using an agentbased framework. This optimization problem is an instant of DSO problems. The general structure of DSO problems is as follows:

$$
\begin{array}{r}
\min _{\boldsymbol{x}_{\mathbf{1}}, \ldots, \boldsymbol{x}_{\boldsymbol{N}}}\left\{\sum_{i=1}^{N} f_{i}\left(\boldsymbol{x}_{\boldsymbol{i}}\right)\right\}, \\
\text { s.t. } \boldsymbol{x}_{\boldsymbol{i}} \in X_{i}
\end{array}
$$

where, $\boldsymbol{x}_{\boldsymbol{i}}$ and $f_{i}$ denote the decision vector and the convex objective function of $i^{t h}$ agent, respectively. Also, $X_{i}$ defines the convex feasible region of vector $x_{i}$. Note that in general, feasible regions and decision vectors of different agents can have overlapping areas and common elements.

Problems of the form (23) can be solved using distributed optimization algorithms without the need for a central solver. In a distributed optimization framework, the agents exchange their estimation of the solution of (23) with each other using a communication network, without sharing their sensitive private cost and feasibility data (i.e., $f_{i}$ and $X_{i}$ ) with their peers. In this paper, we have employed the DGA to solve (22) in a distributed manner. The DGA consists of three main steps that are performed at each iteration of the algorithm:

- Step I: at the $k^{\text {th }}$ iteration of the algorithm, the $i^{\text {th }}$ agent performs a weighted averaging operation (with weights $a_{i}^{j}$ ) over the received signals from its neighboring agents (i.e., including its own estimated solution):

$$
\omega_{i}(k)=\sum_{j=1}^{N_{i}} a_{i}^{j} x_{j}(k)
$$

where, $N_{i}$ denotes the $i^{\text {th }}$ agent's number of neighboring agents (including the $i^{\text {th }}$ agent). In this paper, a uniform weighting mechanism is selected for the distributed optimization model (i.e., $a_{i}^{j}=\frac{1}{N_{i}}$ ). Any weighting mechanism that satisfies the double-stochasticity property (i.e., $\sum_{j=1}^{N_{i}} a_{i}^{j}=1$ and $\sum_{i=1}^{N} a_{i}^{j}=1$ ) guarantees the convergence of the algorithm [24]. A uniform weighting scheme not only satisfies double-stochasticity, but is also intuitive and easy to implement.

- Step II: at this step, each agent performs a gradient descent operation, as follows:

$$
\boldsymbol{v}_{\boldsymbol{i}}(k)=\omega_{\boldsymbol{i}}(k)-\alpha_{k} \cdot \nabla_{\boldsymbol{x}_{\boldsymbol{i}}} f_{i}\left(\boldsymbol{x}_{\boldsymbol{i}}(k)\right)
$$

where, $\alpha_{k}$ is a time-varying weight factor and is selected as $\alpha_{k}=\frac{\gamma}{k+1}$, with $\gamma$ acting as a tunnable parameter of the model.

- Step III: each agent projects the outcome of Step II into its private feasible region to update its estimated solution for the next iteration:

$$
x_{i}(k+1)=\Pi_{X_{i}}\left\{v_{i}(k)\right\}
$$

where, $\Pi_{X_{i}}$ defines the projection operation into the set $X_{i}$. Note that the projection operation is a convex quadratic programming [32], which is determined as follows:

$$
\begin{gathered}
\Pi_{X_{i}}\left\{\boldsymbol{v}_{\boldsymbol{i}}(\boldsymbol{k})\right\}=\underset{\boldsymbol{y}}{\arg \min }\left\|\boldsymbol{y}-\boldsymbol{v}_{\boldsymbol{i}}(\boldsymbol{k})\right\| \\
\text { s.t. } \boldsymbol{y} \in X_{i}
\end{gathered}
$$

where, $\|$.$\| is the Euclidean norm.$

Mathematical properties of the DGA, including the guarantees of convergence, are discussed in [24] and [25] in details, and we refrain from discussing these properties here. However, an interesting practical property of the DGA is that even for time-varying communication networks (e.g., when some communication links are lost temporarily) it is guaranteed to reach the optimal solution, as long as each agent is able to affect every other agent's estimation (directly or indirectly), infinitely often [24].

To apply the DGA to the NBS-based power management problem, we compare (23) and (22), and note that the following holds for each microsource agent in the MG:

$$
f_{i}(\boldsymbol{P})=-\log \left(\prod_{j=1}^{O_{i}}\left(U_{i}^{j}-d_{i}^{j}\right)\right) .
$$

It can be shown that the gradient of $f_{i}(\boldsymbol{P})$ with respect to the vector of decision variables (i.e., $\boldsymbol{P}$ ) can be obtained for each agent, as follows:

$$
\nabla_{\boldsymbol{P}} f_{i}(\boldsymbol{P})=-\left[\begin{array}{ccc}
\frac{\partial U_{i}^{1}}{\partial P_{1}} & \ldots & \frac{\partial U_{i}^{O_{i}}}{\partial P_{1}} \\
\vdots & \ddots & \vdots \\
\frac{\partial U_{i}^{1}}{\partial P_{M}} & \cdots & \frac{\partial U_{i}^{O_{i}}}{\partial P_{M}}
\end{array}\right]\left[\begin{array}{c}
\frac{1}{U_{i}^{1}-d_{i}^{1}} \\
\vdots \\
\frac{1}{U_{i}^{O_{i}}-d_{i}^{O_{i}}}
\end{array}\right]
$$

where, the first term on the right side of (29) is a sensitivity matrix, which is equal to the transpose of the Jacobian matrix of the vector function $\boldsymbol{U}_{\boldsymbol{i}}(\boldsymbol{P})=\left[U_{i}^{1}(\boldsymbol{P}) \ldots U_{i}^{O_{i}}(\boldsymbol{P})\right]^{T}$. The second term in (29) is a vector which embodies the effect of disagreement points of the bargaining. Basically, its function is to push the decision variables away from the disagreement 
region that represents the worst case outcome of the bargaining. Equation (29) is substituted in (25) to complete the distributed bargaining framework for the MG. To guarantee the convergence of the DGA to the optimal solution, the gradient of the global objective function of the optimization problem needs to be defined and finite inside the feasible decision region of the agents [24] [25]. In order to achieve this, in practice the disagreement points of the agents need to be placed outside (but close to) the feasible region of the decision problem to avoid singular gradient values.

The agents participating in the bargaining process, their objective function sets, and feasible regions are as follows:

1) DG agent: $\boldsymbol{U}_{\mathbf{1}}=\left[\begin{array}{ll}U_{1}^{1} & U_{1}^{2}\end{array}\right]^{T}$ and $X_{1}=\{(4) \cap(5) \cap(17)\}$.

2) DRP agent: $\boldsymbol{U}_{\mathbf{2}}=\left[\begin{array}{lll}U_{2}^{1} & U_{2}^{2} & U_{2}^{3}\end{array}\right]^{T}$ and $X_{2}=\{(10) \cap$ $(12) \cap(13) \cap(17)\}$.

3) PCC agent: $\boldsymbol{U}_{\mathbf{3}}=\left[U_{3}^{1}\right]^{T}$ and $X_{3}=\{(16) \cap(17)\}$.

4) ESS agent: $\boldsymbol{U}_{\mathbf{4}}=[0]^{T}$ and $X_{4}=\{(6) \cap(7) \cap(8) \cap(17)\}$.

To summarize, NBS has the following advantages compared to conventional power management approaches: uniqueness, fairness, Pareto-optimality, and the capability to be obtained through a distributed optimization framework.

\section{NuMERICAL EXPERIMENTS AND RESUltS}

The proposed MO bargaining framework is tested on two distinct MGs: 1) a generic islanded MG (Fig. 1), and 2) a modified version of IEEE standard 13-bus distribution network, as a grid-connected MG, including a solar PV farm (Fig. 2). The detailed system and microsource data for these two MGs can be found in [30] and [2], respectively. The fixed load data and the PV power output used in the simulations are adopted from [33], and [34], respectively. The non-controllable fixed load profile and the PV power output for the 13-bus system are shown in Fig. 3 and Fig. 4 (similar curves can be drawn for the islanded MG). Note that, as discussed before, these two curves act as inputs to the model and need to be forecasted for the decision horizon. In this paper we have used a time step of 5 minutes $(\Delta t=5 \mathrm{~min})$ and a look-ahead decision horizon of 4 hours $(T=4$ hours and $H=48)$. The simulation results are obtained under fixed and time-varying (ToU) pricing scenarios.

\section{A. Case I: Islanded $M G$}

To verify the results of the proposed distributed bargaining framework, the optimization problem (22) has also been solved using a central optimization method on a generic islanded $\mathrm{MG}$ (shown in Fig. 1). It was observed that both the distributed optimization framework and the central solver yielded the same results, which confirms the validity of the proposed methodology.

To show that the NBS of the MOPM lies on the Pareto-front of the objective space, we have obtained the Pareto-front of the power management problem of the islanded MG, using a central weighted-sum optimization approach [8]. The problem description for the central weighted-sum optimization to obtain the Pareto-front is as follows:

$$
\begin{aligned}
& \max _{\boldsymbol{P}}\left\{\sum_{i=1}^{N} \sum_{j=1}^{O_{i}} \gamma_{i}^{j} \cdot U_{i}^{j}\right\}, \\
& \sum_{i=1}^{N} \sum_{j=1}^{O_{i}} \gamma_{i}^{j}=1,0 \leq \gamma_{i}^{j} \leq 1, \\
& \text { s.t. (4), (5), (6), (7), (8), (10), (12), (13), (16), (17) }
\end{aligned}
$$

where, the weights $\gamma_{i}^{j}$ are varied to track the Pareto-front of the MO problem. Since this problem is convex then by solving the central optimization (30) the entire Pareto-front can be mapped [8].

In this case, three objectives are considered to be able to show the Pareto-front on a graph (Fig. 5). These objectives are: DG efficiency, curtailable DR utility, and profit (which in an islanded system turns into the negative of cost of production). The NBS of the power management problem is also obtained using the proposed distributed optimization framework and shown on the same figure. As can be seen in Fig. 5, the NBS (obtained through distributed optimization) is correctly located on the Pareto-front of the power management problem (obtained using a central optimization technique), as was expected. This confirms that the distributed optimization framework converges to a solution on the Pareto-front of the MOPM problem. This solution, as discussed before, is the NBS of the bargaining game. We have used Case I as a result verification tool.

\section{B. Case II: Grid-Connected MG}

In this section, we present the results of the simulations on the grid-connected MG, shown in Fig. 2. All the six objective functions considered in Section II are taken into account. The unit price of exchanged energy with the main grid (fixed and ToU pricing) are shown in Fig. 6.

In Fig.7, the output power of the thermal DG unit is shown. As can be seen, the output power of the DG is almost the same under both of the pricing scenarios. Also, due to the introduced ramp rate constraint (5) the DG needs to start increasing its output power at an earlier time to be able to respond to the peak load, at the later time of the day. On the other hand, the effect of variable energy pricing is notably observed in the behavior of time-shiftable DR resources, shown in Fig. 8. As can be seen in this figure, compared to the fixed pricing scenario (original scheduled profile), under ToU pricing the time-shiftable DR resources are deployed in a way to eliminate consumption during peak-price time period and reduce the cost of consumption (note that under both pricing scenarios the same amount of energy is consumed in a pre-defined time frame). The original available power value of time-shiftable loads is shown in the figure under fixed price scenario. These resources are available from time 18:00 to 21:00, and can be shifted within the time interval 16:00 to 23:00 (i.e., they cannot be consumed before 16:00 or after 23:00). Since in the fixed price scenario the time-shiftable loads cannot achieve any cost savings by shifting their consumption (due to the fixed price), 


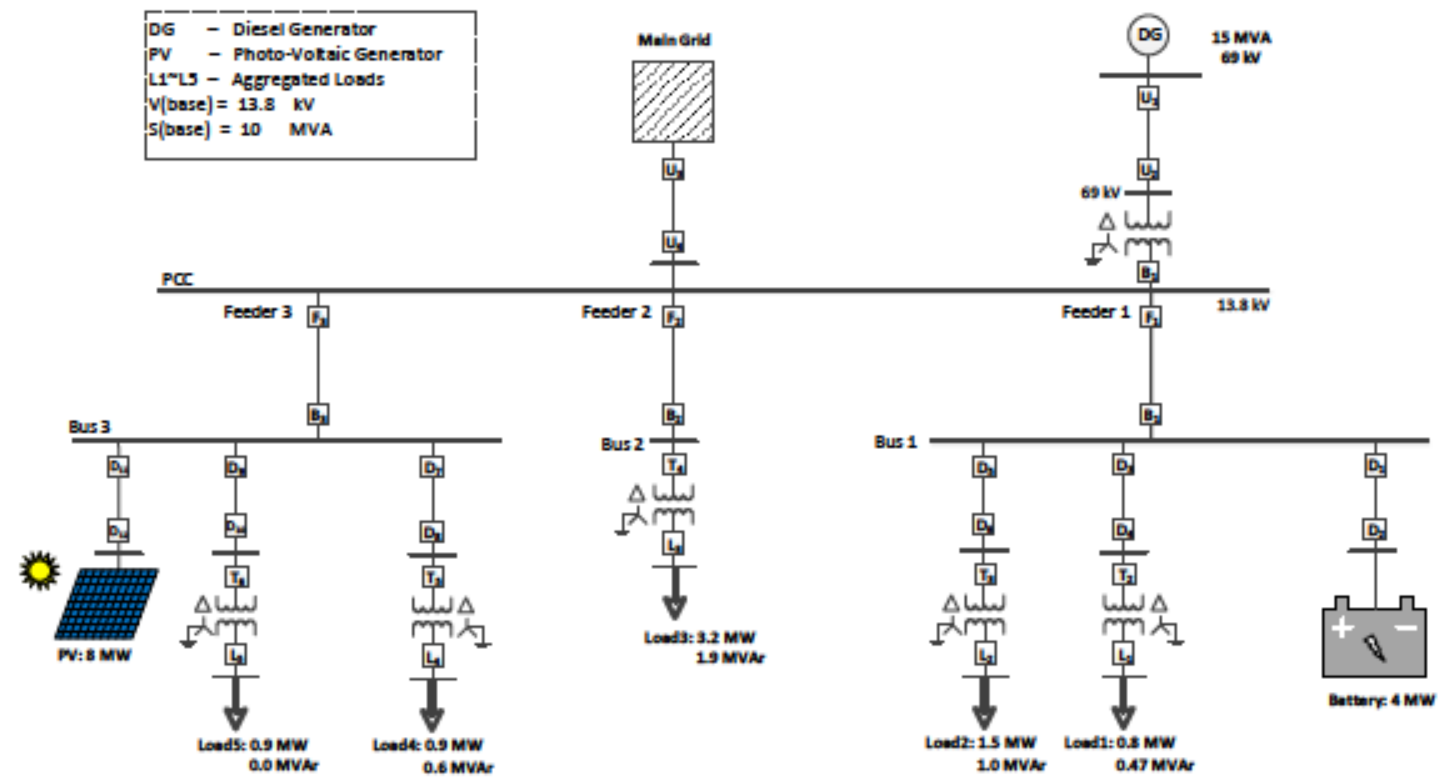

Fig. 2. Modified IEEE 13-bus standard system, as a grid-connected MG.

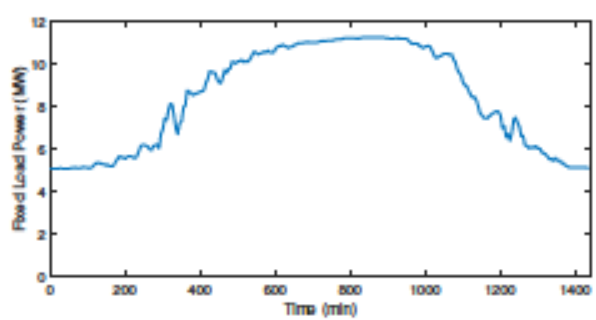

Fig. 3. Uncontrollable fixed load profile.

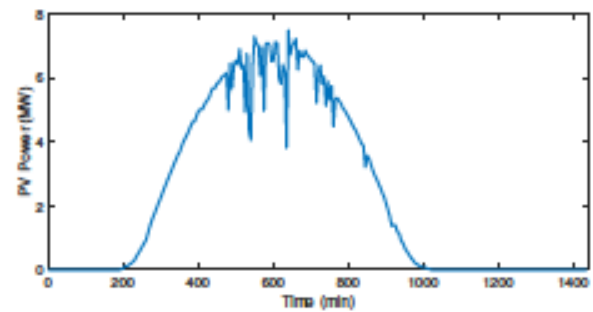

Fig. 4. Output power of PV power generator.

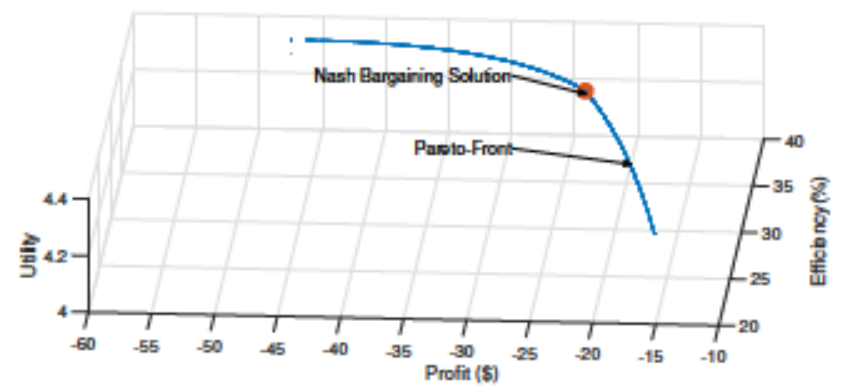

Fig. 5. The Pareto-front and the NBS. no load shifting is performed and the consumers fulfill their original schedule (which has a peak of around $2 \mathrm{MW}$, used as an input to the decision model).

The effect of time-shiftable load can be observed in the power exchange with the main grid, shown in Fig. 9. As can be seen in this figure, due to the load-shifting process, the amount of power export to the main grid is increased considerably during the peak-price period, to gain more profit from the sale of energy. This comes at the expense of lower power export at earlier times. Note that because of the objective function (15), the exported power is limited to $4.2 \mathrm{MW}$ (Fig. 9) and does not reach its congestion limit of 7.5 MW. However, when objective function (15) is removed the exported power to the grid is set to its maximum feasible value of 7.5 MW for most of the time (except for a short period before price peak interval, where time-shiftable loads create an increase in power consumption which leads to less power export).

The profit curve of the MG is shown in Fig. 10, where we observe a $47 \%$ increase in the total profit level of the MG, under ToU pricing with time-shiftable DR resources. Also, compared to the case of ToU pricing without timeshiftable DR resources, the total profit level shows $17.6 \%$ improvement. Without objective function (15), higher export levels lead to considerably higher profit, as shown in the figure. However, this comes at the expense of high power utilization and congestion of the line at the PCC. Since line congestion on the lines signal the need for network expansion/upgrade, by using objective function (15) we are creating a balance between the two objectives: short-term profit and avoiding the need for long-term expansion of distribution system.

The total cost of power consumption is shown in Fig. 11. As can be seen in this figure, the cost of consumption increases at the times where the energy price is higher. However, the total cost of consumption is reduced by $44 \%$ when time-shiftable 


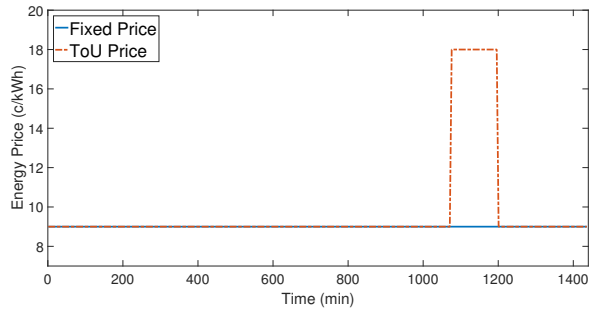

Fig. 6. Main grid energy price.

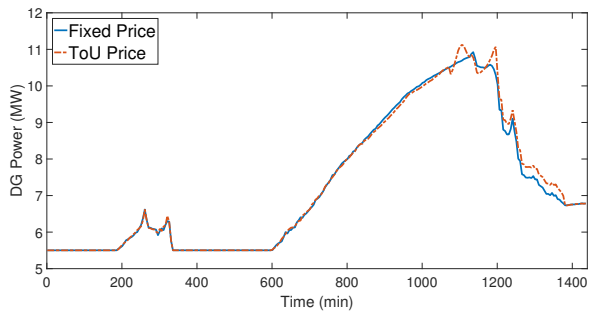

Fig. 7. DG output power.

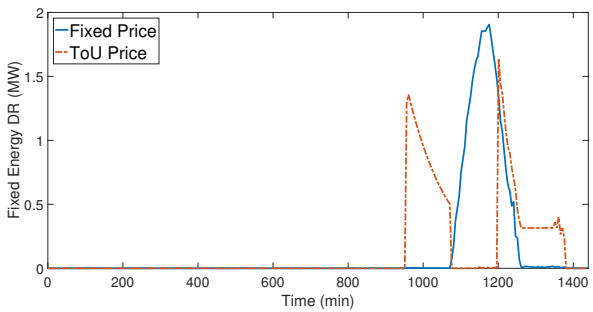

Fig. 8. Time-shiftable DR resource profiles.

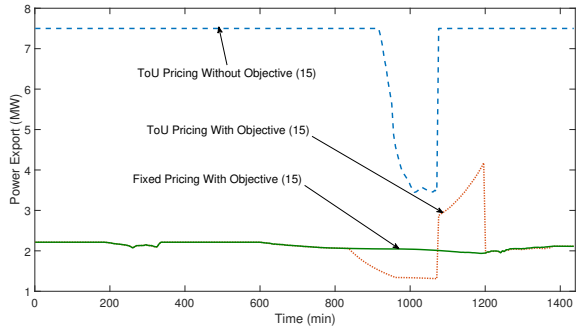

Fig. 9. Exchanged power with the grid.

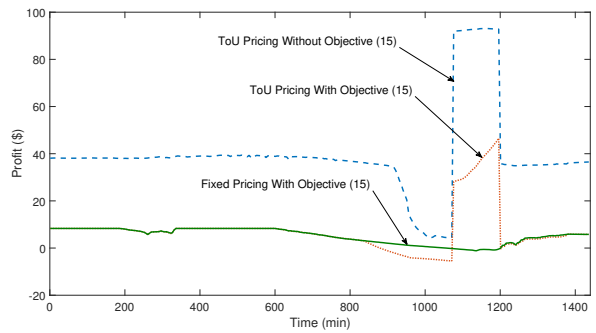

Fig. 10. Profit profile of the MG.

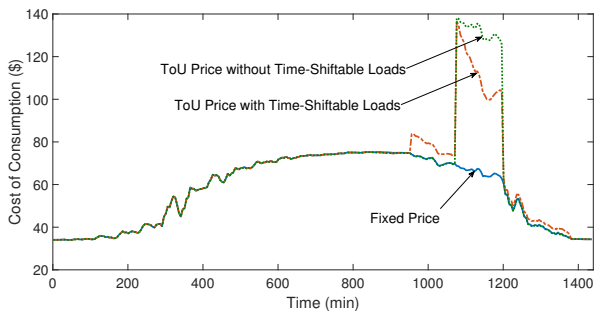

Fig. 11. Total cost of power consumption.

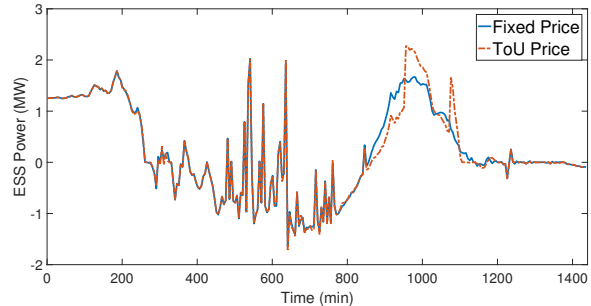

Fig. 12. Battery storage output power.

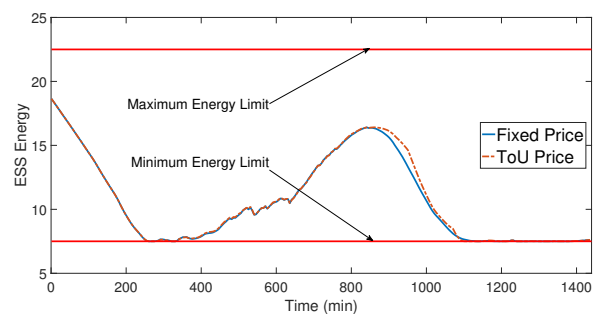

Fig. 13. Stored energy profile of the battery system.

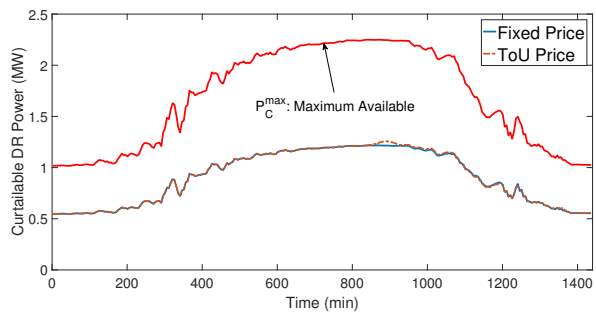

Fig. 14. Curtailable DR power profile.

loads are present, compared to the case without time-shiftable loads.

The power and stored energy of the battery system are depicted in Fig. 12 and Fig. 13, respectively (with negative power values indicating battery charging). As is observed in these figures the volatility and variations of the renewable PV power is absorbed by the storage system. Hence, from the perspective of the main grid, the volatility of the PV power does not affect the grid (note the smooth power curve of Fig. 9). This implies that employing the proposed MGbased power management procedure, the undesired effects of volatility of distributed renewable resources can be limited, which leads to the successful integration of these resources into the electrical energy networks. Also, it can be observed from Fig. 13 that the stored energy of the battery is correctly kept between its maximum and minimum limits corresponding to $S O C^{\max }=0.9$, and $S O C^{\min }=0.3$, respectively.

In Fig. 14, the maximum available power profile of curtailable DR resources and the portion used in our proposed method are shown (for $\omega=3$ ). The maximum available curtailable load is assumed to be equal to $20 \%$ of total fixed load at all times. The percentage of the DR employed under our proposed method (for both ToU and fixed price scenarios) is only $50 \%$ of the maximum available curtailable DR resources. 


\section{CONCLUSION}

In this paper a bargaining framework was proposed to solve the MOPM problem of MGs. The proposed framework employs NBS to find a unique and fair solution on the Paretofront of the optimization problem. Moreover, the solution is obtained using a distributed optimization method which relies on an agent-based decision architecture. It is shown that using the proposed methodology the MOPM problem can be solved in islanded and grid-connected MGs with various types of microsources, including DGs, renewable power generators, ESS units, and curtailable and time-shiftable loads.

\section{REFERENCES}

[1] S. Chowdhury and P. Crossley, Microgrids and active distribution networks. Stevenage: The Institution of Engineering and Technology, 2009.

[2] A. Pourmousavi and M. H. Nehrir, "Real-time central demand response for primary frequency regulation in microgrids," IEEE Trans. Smart Grid, vol. 3, no. 4, pp. 1988-1996, Dec. 2012.

[3] A. Khodaei, "Resiliency-oriented microgrid optimal scheduling," IEEE Trans. Smart Grid, vol. 5, no. 4, pp. 1584-1591, Jul. 2014.

[4] J. M. Guerrero, J. C. Vasquez, J. Matas, L. G. de Vecuna, and M. Castilla, "Hierarchical control of droop-controlled ac and dc microgrids - a general approach toward standardization," IEEE Trans. Ind. Electron., vol. 58, no. 1, pp. 158-172, Jan. 2011.

[5] A. Klem, H. Nehrir, and K. Dehghanpour, "Frequency stabilization of an islanded microgrid using droop control and demand response," In Proceedings, North American Power Symposium (NAPS), pp. 1-6, 2016.

[6] R. P. Behnke, C. Benavides, F. Lanas, B. Severino, L. Reyes, J. Llanos, and D. Saez, "A microgrid energy management system based on the rolling horizon strategy," IEEE Trans. Smart Grid, vol. 4, no. 2, pp. 996-1006, Jun. 2013.

[7] H. Nafisi, M. M. Agha, H. A. Abyaneh, and M. Abedi, "Two-stage optimization method for energy loss minimization in microgrid based on smart power management scheme of phevs," IEEE Trans. Smart Grid, vol. 7, no. 3, pp. 1268-1276, May 2016.

[8] K. Deb, Multi-objective optimization using evolutionary algorithms. West Sussex: John Wiely \& Sons, 2001.

[9] A. Chaouachi, R. M. Kamel, R. Andoulsi, and K. Nagasaka, "Multiobjective intelligent energy management for a microgrid," IEEE Trans. Ind Electron., vol. 60, no. 4, pp. 1688-1699, Apr. 2013.

[10] B. Zhao, X. Zhang, J. Chen, C. Wang, and L. Guo, "Operation optimization of standalone microgrids considering lifetime characteristics of battery energy storage system," IEEE Trans. Sustain. Energy, vol. 4, no. 4, pp. 934-943, Oct. 2013.

[11] M. Ross, C. Abbey, F. Bouffard, and G. Joos, "Multiobjective optimization dispatch for microgrids with a high penetration of renewable generation," IEEE Trans. Sustain. Energy, vol. 6, no. 4, pp. 1306-1314, Oct. 2015.

[12] C. M. Colson, M. H. Nehrir, R. K. Sharma, and B. Asghari, "Improving sustainability of hybrid energy systems part ii: managing multiple objectives with a multiagent system," IEEE Trans. Sustain. Energy, vol. 5, no. 1, pp. 46-54, Jan. 2014.

[13] P. Li, J. Ma, and B. Zhao, "Game theory method for multiobjective optimal operation of microgrid," IEEE Power and Energy General Meeting, pp. 1-5, 2015.

[14] M. Maschler, E. Solan, and S. Zamir, Game theory. New York: Cambridge University Press, 2013.

[15] Z. Zhang, J. Shi, H. H. Chen, M. Guizani, and P. Qui, "A cooperation strategy based on nash bargaining solution in cooperative relay networks," IEEE Trans. Veh. Technol., vol. 57, no. 4, pp. 2570-2577, Jul. 2008.

[16] Z. Han, Z. Ji, , and K. J. R. Liu, "Fair multiuser channel allocation for OFDMA networks using nash bargaining solutions and coalitions," IEEE Trans. Commun., vol. 53, no. 8, pp. 1366-1376, Aug. 2005.

[17] H. Park and M. Schaar, "Bargaining strategies for networked multimedia resource management," IEEE Trans. Signal Process., vol. 55, no. 7, pp. 3496-3511, Jul. 2007.

[18] S. Kishimoto and S. Muto, "Fee versus royalty policy in licensing through bargaining: an application of the nash bargaining solution," Bulletin of Economic Research, vol. 64, no. 2, pp. 293-304, Apr. 2012.
[19] A. Kampas and B. White, "Selecting permit allocation rules for agricultural pollution control: a bargaining solution," Ecological Economics, vol. 47, no. 2-3, pp. 135-147, Dec. 2003.

[20] P. A. Chiappori, B. Fortin, and G. Lacroix, "Marriage market, divorce legislation, and household labor supply," Journal of political Economy, vol. 110, no. 1, pp. 37-72, Feb. 2002.

[21] G. Shrimali, A. Akella, and A. Mutapcic, "Cooperative interdomain traffic engineering using nash bargaining and decomposition," IEEE/ACM Trans. Netw., vol. 18, no. 2, pp. 341-352, Apr. 2010.

[22] K. Dehghanpour and H. Nehrir, "Intelligent microgrid power management using the concept of Nash bargaining solution," Submitted to 2017 Intelligent Systems Application to Power systems (ISAP), pp. 1-6, 2017.

[23] I. Necoara, V. Nedelcu, and I. Dumitrache, "Parallel and distributed optimization methods for estimation and control networks," Journal of Process Control, vol. 21, no. 5, pp. 756-766, Jun. 2011.

[24] A. Nedic and A. Ozdaglar, "Distributed subgradient methods for multiagent optimization," IEEE Trans. Automat. Control, vol. 54, no. 1, pp. 48-61, Jan. 2009.

[25] A. Nedic, A. Ozdaglar, and P. A. Parrilo, "Constrained consensus and optimization in multi-agent networks," IEEE Trans. Automat. Control, vol. 55, no. 4, pp. 922-938, Apr. 2010.

[26] W. Zhang, W. Liu, X. Wang, L. Liu, and F. Ferrese, "Online optimal generation control based on constrained distributed gradient algorithm," IEEE Trans. Power Syst., vol. 30, no. 1, pp. 35-45, Jan. 2015.

[27] "European Information \& Communication Technology Association (EICTA): Interoperability white paper - Jun. 2004," http://agoria.be/ www1.wsc/webextra/prg/.

[28] C. Yang, A. A. Thatte, and L. Xie, "Multitime-scale data-driven spatiotemporal forecast of photovoltaic generation," IEEE Trans. Sustain. Energy, vol. 6, no. 1, pp. 104-112, Jan. 2015.

[29] C. Guan, P. B. Luh, L. D. Michel, Y. Wang, and P. B. Friedland, "Very short-term load forecasting: wavelet neural networks with data pre-filtering," IEEE Trans. Power Syst., vol. 28, no. 1, pp. 30-41, Feb. 2013.

[30] A. Pourmousavi, M. H. Nehrir, and R. K. Sharma, "Multi-timescale power management for islanded microgrids including storage and demand response," IEEE Trans. Smart Grid, vol. 6, no. 3, pp. 1185-1195, May 2015.

[31] Z. M. Fadlullah, D. M. Quan, N. Kato, and I. Stojmenovic, "GTES: an optimized game-theoretic demand-side management scheme for smart grid," IEEE Syst. J., vol. 8, no. 2, pp. 588-597, Jun. 2014.

[32] S. Boyd and N. Vandenberghe, Convex optimization. New York: Cambridge University Press, 2009.

[33] "Lawrence berkeley national laboratory: the carbon dioxide abatement potential of california's mid-sized commercial buildings - Jan. 2010.”

[34] "Electric Power Research Institute (EPRI): Distributed PV monitoring and feeder analysis - Jun. 2012," http://dpv.epri.com/measurement_data. html.

Kaveh Dehghanpour (S'14) received the B.S. and M.S. degrees from the University of Tehran, Tehran, Iran, in 2011, and 2013, respectively. $\mathrm{He}$ is currently pursuing the Ph.D. degree in the Electrical and Computer Engineering Department at Montana State University, Bozeman, MT, USA. His research interests include electrical energy markets, market-based control, agent-based modeling, and machine learning.

Hashem Nehrir (S'68-M'71-SM'89-F'10-LF'13) received the B.S., M.S., and Ph.D. degrees from Oregon State University, Corvallis, OR, USA, in 1969, 1971, and 1978, respectively, all in electrical engineering. He is a Professor in the Department of Electrical and Computer Engineering, Montana State University, Bozeman, MT, USA. His research interests include modeling and control of power systems, alternative energy power generation systems, and application of intelligent controls to power systems. He is the author of three textbooks and an author or coauthor of numerous technical papers. 